\author{
Gabriele DI LORENZO ${ }^{1}$ \\ Maria Luisa PACOR ${ }^{2}$ \\ Giuseppina CANDORE ${ }^{3}$ \\ Florinda LISTI ${ }^{3}$ \\ Vito DITTA ${ }^{1}$ \\ Maria Stefania LETO-BARONE ${ }^{1}$ \\ Alberto D'ALCAMO ${ }^{1}$ \\ Nicola MARTINELLI ${ }^{2}$ \\ Roberto CORROCHER ${ }^{2}$ \\ Calogero CARUSO ${ }^{3}$
}

${ }^{1}$ Dipartimento di Medicina Clinica e delle
Patologie Emergenti,
Università di Palermo, Via del Vespro,
141-90127 Palermo, Italy
${ }^{2}$ Dipartimento di Medicina Clinica e
Medicina Sperimentale,
Università di Verona,
Policlinico G.B. Rossi, Università di Verona,
Piazzale Scuro, Verona 10-37134, Italy
${ }^{3}$ Dipartimento di Biopatologia e
Metodologie Biomediche,
Università di Palermo,
Istituto di Patologia generale,
Corso Tukory 211-90134 Palermo, Italy

Reprints: G. Di Lorenzo

<dilo601@unipa.it>

\section{Polymorphisms of cyclo-oxygenases and 5-lipo-oxygenase-activating protein are associated with chronic spontaneous urticaria and urinary leukotriene $\mathrm{E} 4$}

\begin{abstract}
Background. The mechanisms of chronic spontaneous urticaria (CSU) continue to be unknown.

Objects. Our working hypothesis is that polymorphisms of cyclooxygenases and 5-lipo-oxygenase-activating protein may be involved in the pathways leading to CSU.

Methods. We examined five candidate polymorphisms of cyclooxygenases 1 and 2 and of 5-lipo-oxygenase-activating protein in 109 controls and in $94 \mathrm{CSU}$ patients from Northern Italy. We also examined the levels of urinary leukotriene E4 (LTE4) before and after challenge with ASA.

Results. A multiple regression model was found to show that COX-2 5'UTR T/G, COX-2 Exon 10 T/C, and FLAP -336 G/A polymorphisms were significantly associated with CSU, with the minor allele more represented in CSU group. Similar results were obtained as regards the specific association with ASA-tolerated CSU and ASA-exacerbated CSU. Evaluating a polygenic model, reflecting the sum of the concomitant alleles associated with CSU (i.e. COX-2 5'UTR G allele, COX-2 Exon $10 \mathrm{C}$ allele, and FLAP -336 G/A allele), the proportion of CSU patients increased progressively with the increasing number of unfavourable alleles. Finally, in a linear regression model after adjustment for disease status COX-1 $22 \mathrm{~T}$ carriership remained a significant predictor of post-challenge high urinary LTE4 levels.

Conclusions. Our results support the hypothesis that polymorphisms of cyclo-oxygenases and 5-lipo-oxygenase-activating protein may be associated with CSU.
\end{abstract}

Key words: chronic spontaneous urticaria, hypersensivity to aspirin, cyclo-oxygenases, 5-lipo-oxygenase-activating protein, urinary leukotriene E4

\begin{abstract}
$\mathrm{A}$ rachidonic acid, released from the mammalian cell membrane, may be metabolized through two main pathways: the cyclooxygenase (COX) pathway to yielding prostanoids or the 5-lipo-oxygenase (5-LO) pathway to form the unstable intermediary LTA4, which, depending on distal enzymes differentially expressed in leukocytes, is metabolized to either LTB4 or the cysteinyl leukotrienes (CysLTs). These mediators are involved in a variety of clinically important areas such as inflammation, fever, thrombosis, allergic and immune responses, and cancer. Acetylsalicylic acid (ASA) causes a shift in the arachidonic acid metabolism away from the cyclooxygenase (COX) pathway toward the lipo-oxygenase (LO) pathways [1]. ASA-induced asthma (AIA) is thought to be caused by a shift in the arachidonic acid metabolism pathaway. The identification of genetic factors that confer susceptibility to the development of acetylsalicylic acid (ASA) exacerbated chronic spontaneous urticaria (AeCSU) could contribute to the understanding of the disease [2]. In
\end{abstract}

contrast with AIA, progress in understanding ASA-induced urticaria has been slow. Clinicians are well aware that up to $20 \%$ to $40 \%$ of patients with chronic spontaneous urticaria (CSU) develop an increase in wheals and swelling after ASA. It was reported that, in some of them, not only ASA but also several other nonsteroidal anti-inflammatory drugs (NSAIDs) inhibit COX triggered skin eruptions [3]. Recent studies on eicosanoid metabolism showed that coxibs, selective COX-2 inhibitors, did not precipitate wheals and swelling in patients with chronic urticaria who were sensitive to aspirin [4-6]. It was also shown that AeCSU is associated with overproduction of cysteinyl-leukotrienes (cys-LTs), as reflected by increased urinary excretion of leukotriene E4 (LTE4) [7, 8]. However, when we compared urinary LTE4 levels in the same subjects, we found no difference at baseline between the groups before challenge with ASA; we found an increase in leukotriene levels after challenge but only in patients with CSU [7]. These results could explain the different clinical responses to therapy with 
Table 1. Clinical characteristic of the study population divided in subgroups according to clinical diagnosis (i.e. normal controls (NC) and patients with chronic spontaneous urticaria (CSU). The latter group was further divided in 2 subgroups: ASA tolerated chronic idiopathic urticaria (AtCSU) and ASA exacerbated chronic urticaria (AeCSU)).

\begin{tabular}{|lllll|}
\hline & $\begin{array}{l}\text { NC } \\
(\mathbf{n = 1 0 9})\end{array}$ & $\begin{array}{l}\text { CSU } \\
(\mathbf{n = 9 4 )}\end{array}$ & $\begin{array}{l}\text { AtCSU } \\
(\mathbf{n = 3 1})\end{array}$ & $\begin{array}{l}\text { AeCSU } \\
(\mathbf{n = 6 3})\end{array}$ \\
\hline $\begin{array}{l}\text { Age } \\
\text { years })\end{array}$ & $36.5 \pm 13.8$ & $39.2 \pm 13.2$ & $39.6 \pm 13.3$ & $39.0 \pm 13.3$ \\
\hline $\begin{array}{l}\text { Males } \\
\text { n } \%)\end{array}$ & $33(30.3)$ & $24(25.5)$ & $4(12.9)^{*}$ & $20(31.7)$ \\
\hline $\begin{array}{l}\text { Positive ASA challenge } \\
\text { n }(\%)\end{array}$ & $0(0)$ & $63(67.0)$ & $0(0)$ & $63(100)$ \\
\hline LTE4 levels before challenge with ASA & $15.7 \pm 1.08$ & & $15.5 \pm 2.05$ & $16.0 \pm 1.1$ \\
\hline LTE4 levels after challenge with ASA & $15.2 \pm 2.1$ & & $14.9 \pm 2.1$ & $69.5 \pm 10.4 \S$ \\
\hline
\end{tabular}

$* P=0.049$ by $\chi^{2}$-test versus AeCSU

$\S_{P}<0.001$ by $t$-test versus both $N C$ and AtCSU

leukotriene receptor antagonist (LT-RAs) in patients with CSU with or without well-defined triggers, as we observed in two placebo-controlled trials with antileukotriene $[9,10]$. The aim of this study was to investigate in a case-control setting the potential association of 5 candidate polymorphisms of genes of cyclo-oxygenases 1 and 2 and of 5-lipo-oxygenase-activating protein $(\mathrm{COX}-122 \mathrm{C} / \mathrm{T}, \mathrm{COX}-$ 2 -162C/G, COX-2 5'UTR T/G, COX-2 Exon 10 T/C, and FLAP -336 G/A) with CSU, as well as with urinary LTE4 levels, both basal and after challenge with ASA. Patients with CSU were further divided into ASA-tolerated $\mathrm{CSU}$ (AtCSU) and AeCSU subgroups and analyzed separately.

\section{Methods}

\section{Patients}

Ninety-four patients with CSU, 24 male and 70 female; mean age: 39.2 yrs (range 18-71 years), were randomly selected by chart review from outpatients of the Dipartimento di Medicina Clinica e Sperimentale of Verona (Italy). All the patients had been previously submitted to oral challenge double-blind placebo-controlled (DBPC) for the ASA using three capsules of placebo (talc) and 10, 10, $20 \mathrm{mg}$ graded doses in brackets. The diagnosis of ASA exacerbation (AeCSU) or ASA-tolerated CSU (AtCSU) was

\author{
Abbreviations: \\ CSU chronic spontaneous urticaria \\ ASA aspirin \\ NSAID nonsteroidal anti-inflammatory drugs \\ AHCU aspirin-hypersensitivity chronic urticaria \\ NAHCU chronic urticaria with negative response to aspirin \\ Cys-LT cysteinyl-leukotrienes \\ LTE4 leukotriene E4 \\ COXs cyclo-oxygenases \\ FLAP 5-lipo-oxygenase-activating protein \\ SNP single nucleotide polymorphisms \\ LTRA LT receptor antagonist \\ NC normal healthy controls \\ PCR polymerase chain reaction \\ HWE Hardy-Weinberg equilibrium
}

based on a positive response or a negative response to ASA challenge, respectively [7]. Of these, 63 were AeCSU (67\%) and 31 were AtCSU, respectively.

A total of 109 normal healthy controls (NC) (33 males and 76 females; mean age: 36.5 yrs (range 18-71 years)) were randomly selected from the pool of healthy volunteers who come to Dipartimento di Medicina Clinica e Sperimentale of Verona for a routine medical examination or who work at the clinic. NC had no history of urticaria or asthmatic or rhinitic symptoms or aspirin hyper-responsiveness. All the NC performed an oral challenge DBPC with ASA which resulted negative in all subjects. The demographic characteristics of patients and the NC are reported in table 1 . We used R.2.7.1 (http://www.r-project.org/) for random selection of participants. All the participants were of the same ethnic background (Caucasians). In all subjects, renal function (serum and urinary creatinine, clearance of creatinine and bun) was normal.

\section{Study protocol}

Based on the results of previous challenges to ASA (see above) the AeCSU were re-challenged with a single dose of ASA, precisely with that dose which had induced urticaria on previous challenge with ASA, and with placebo. AtCSU patients and $\mathrm{NC}$ were re-challenged with $20 \mathrm{mg}$ of ASA and placebo.

The AeCSU patients were re-challenged twice, on different days, with ASA which have induced urticaria to previous challenge with ASA and with placebo. The AtCSU patients and NC were re-challenged twice with $20 \mathrm{mg}$ of ASA and with placebo. None of the patients showed significant cutaneous symptoms at the time of testing. Antihistamines were interrupted at least 8 days before the challenges, and no drugs were used within $48 \mathrm{~h}$ of testing. No patient had taken oral corticosteroids.

All the AeCSU patients presented the onset of urticaria (weal and erythema) after 30-45 min. after the ingestion of ASA. None of the AtCSU and NC presented urticaria with $20 \mathrm{mg}$ of ASA. None of the subjects studied presented urticaria after placebo.

\section{$\mathrm{LTE}_{\mathbf{4}}$ determination in urine}

Urinary LTE $_{4}$ was measured before and after ASA challenge. Measurements were made at same time, in duplicate, 
and expressed as nanograms per milligram of creatinine. $\mathrm{LTE}_{4}$ determination in urine was performed exactly as described by Di Lorenzo et al. [7-11].

\section{Molecular methods}

The method used to extract the DNA was salting-out, following the standard protocol [12]. The DNA samples were amplified for $-336 \mathrm{G} / \mathrm{A}$ polymorphism of FLAP gene by a polymerase chain reaction (PCR). Concerning the $22 \mathrm{C} / \mathrm{T}$ of $\mathrm{COX}-1$ gene and $-162 \mathrm{C} / \mathrm{G}, 5^{\prime} \mathrm{UTR} \mathrm{T} / \mathrm{G}$, and exon10 T/C polymorphisms of COX-2 gene, DNA samples were analyzed by PCR-SSP, using a PCR thermal sequencer (MyCycler Biorad, Milan, Italy). The products were obtained after amplification in a $20 \mu \mathrm{L}$ volume containing $20 \mathrm{ng}$ of genomic DNA template, $1 \mathrm{U}$ of Taq DNA polymerase, $200 \mu \mathrm{M}$ dNTP, $1 \mathrm{X}$ buffer containing $(10 \mathrm{mmol} / \mathrm{L}$ Tris- $\mathrm{HCl} \mathrm{pH} \mathrm{8.3,50} \mathrm{mmol/L} \mathrm{KCl}), 1.3 \mathrm{mmol} / \mathrm{L}$ $\mathrm{MgCl}_{2}$, and $0.2 \mathrm{pmol} / \mathrm{L}$ of each amplification primer.

\section{Statistical analysis}

Calculations were performed with the SPSS 17.0 statistical package (SPSS Inc., Chicago, IL). Distributions of continuous variables in groups were expressed as means \pm standard deviation. Quantitative data were assessed using the Student's t-test or by ANOVA. Qualitative data were analyzed with the $\chi^{2}$-test. Considering the low frequency of minor allele homozygotes, most analyses were performed comparing the carriers of the minor allele (heterozygotes + homozygotes) with major allele homozygotes. A value of $\mathrm{P}<0.05$ was considered statistically significant. Hardy-Weinberg equilibrium (HWE) was tested for each genotype within the control group by means of $\chi^{2}$-test. To assess the extent to which gene polymorphisms were associated with CSU (as well as with AtCSU and with AeCSU), odds ratios with $95 \%$ CIs were estimated by a sex- and age-adjusted logistic regression analysis. A logistic regression model was also used to analyze potential interactions among gene polymorphisms in predicting CSU risk. Then we focused on a polygenic additive model. On this basis, we attributed to each patient a "score", reflecting the sum of the "unfavourable" CSU-associated alleles, theoretically ranging from 0 (no risk allele present) to 6 (all the risk alleles present). The association between this score and CSU was evaluated by $\chi^{2}$ for linear trend analysis.

Finally, a linear regression analysis adjusted for sex, age, and disease status was performed to assess which polymorphisms were independent predictors of urinary LTE4 levels variability.

The study was approved by Ethical Committee of Verona and written informed consent to the study was obtained from each patient. The study was performed following the Helsinki Declaration of 1975, as amended in 1983.

Table 2. Genotypic distributions of COX-1, COX-2, and FLAP polymorphisms in the study population divided in subgroups according to clinical diagnosis (i.e. normal controls (NC) and patients with chronic spontaneous urticaria (CSU). The latter group was further divided in 2 subgroups: ASA tolerated chronic spontaneous urticaria (AtCSU) and ASA exacerbated chronic spontaneous urticaria (AeCSU).

\begin{tabular}{|c|c|c|c|c|c|c|c|c|c|c|}
\hline Gene & SNP & Genotype & $\begin{array}{l}N C \\
(n=109)\end{array}$ & $\begin{array}{l}\text { CSU } \\
(n=94)\end{array}$ & $\begin{array}{l}\text { NC vs NC } \\
\text { vs CSU } \\
P *\end{array}$ & $\begin{array}{l}\text { AtCSU } \\
(n=31)\end{array}$ & $\begin{array}{l}\text { NC vs } \\
\text { AtCSU } \\
P *\end{array}$ & $\begin{array}{l}\text { AeCSU } \\
(n=63)\end{array}$ & $\begin{array}{l}\text { NC vs } \\
\text { AeCSU } \\
P *\end{array}$ & $\begin{array}{l}\text { AeCSU vs } \\
\text { AtCSU } \\
P^{*}\end{array}$ \\
\hline \multirow[t]{4}{*}{ COX-1 } & 22 & $\mathrm{CC}$ & $100(91.7)$ & $73(77.7)$ & & $24(77.4)$ & & $49(77.8)$ & & \\
\hline & $\mathrm{C} / \mathrm{T}$ & CT & $8(7.4)$ & $15(16.0)$ & 0.012 & $5(16.1)$ & 0.049 & $10(15.9)$ & 0.021 & 0.999 \\
\hline & $\mathrm{n}(\%)$ & TT & $1(0.9)$ & $6(6.3)$ & & $2(6.5)$ & & $4(6.3)$ & & \\
\hline & & $\mathrm{T}$ carrier & $9(8.3)$ & $21(22.3)$ & 0.005 & $7(22.6)$ & 0.027 & $14(22.2)$ & 0.010 & 0.969 \\
\hline \multirow[t]{12}{*}{$\mathrm{COX}-2$} & -162 & $\mathrm{CC}$ & $39(35.8)$ & $29(30.9)$ & & $10(32.3)$ & & $19(30.2)$ & & \\
\hline & $\mathbf{C} / \mathbf{G}$ & CG & $57(52.3)$ & $52(55.3)$ & 0.743 & 16(51.6) & 0.811 & $36(57.1)$ & 0.753 & 0.852 \\
\hline & $\mathrm{n}(\%)$ & GG & $13(11.9)$ & $13(13.8)$ & & $5(16.1)$ & & $8(12.7)$ & & \\
\hline & & G carrier & $70(64.2)$ & $65(69.1)$ & 0.458 & $21(67.7)$ & 0.717 & $44(69.8)$ & 0.452 & 0.836 \\
\hline & $5^{\prime}$ UTR & TT & $103(94.5)$ & $67(71.3)$ & & $23(74.2)$ & & $44(69.8)$ & & \\
\hline & T/G & TG & $6(5.5)$ & $16(17.0)$ & $<0.001$ & $4(12.9)$ & $<0.001$ & $12(19.1)$ & $<0.001$ & 0.751 \\
\hline & $\mathrm{n}(\%)$ & GG & $0(0)$ & $11(11.7)$ & & $4(12.9)$ & & $7(11.1)$ & & \\
\hline & & G carrier & $6(5.5)$ & $27(28.7)$ & $<0.001$ & $8(25.8)$ & 0.001 & $19(30.2)$ & $<0.001$ & 0.661 \\
\hline & Exon 10 & TT & $98(89.9)$ & $72(76.6)$ & & $21(67.7)$ & & $51(81.0)$ & & \\
\hline & $\mathrm{T} / \mathrm{C}$ & TC & $10(9.2)$ & $20(21.3)$ & 0.037 & $9(29.0)$ & 0.010 & $11(17.5)$ & 0.250 & 0.360 \\
\hline & $\mathrm{n}(\%)$ & $\mathrm{CC}$ & $1(0.9)$ & $2(2.1)$ & & $1(3.3)$ & & $1(1.5)$ & & \\
\hline & & $\mathrm{C}$ carrier & $11(10.1)$ & $22(23.4)$ & 0.010 & $10(32.3)$ & 0.002 & $12(19.0)$ & 0.096 & 0.155 \\
\hline \multirow[t]{4}{*}{ FLAP } & -336 & GG & $99(90.8)$ & $60(63.8)$ & & $17(54.8)$ & & $43(68.3)$ & & \\
\hline & G/A & GA & $10(9.2)$ & $29(30.9)$ & $<0.001$ & $10(32.3)$ & $<0.001$ & $19(30.2)$ & 0.001 & 0.060 \\
\hline & $\mathrm{n}(\%)$ & $\mathrm{AA}$ & $0(0)$ & $5(5.3)$ & & $4(12.9)$ & & $1(1.5)$ & & \\
\hline & & A carrier & $10(9.2)$ & $34(36.2)$ & $<0.001$ & $14(45.2)$ & $<0.001$ & $20(31.7)$ & $<0.001$ & 0.203 \\
\hline
\end{tabular}

*by $\chi^{2}$-test 


\section{Results}

The clinical and laboratory characteristics of the study population are summarized in table 1. Males were slightly less represented in the AtCSU. As expected, patients with AeCSU had higher levels of LTE4 after challenge with ASA than either NC or AtCSU, whereas no difference was found for LTE4 basal levels (table 1).

The genotype frequencies in the study population according to clinical diagnosis are shown in table 2. All the analysed SNPs were in Hardy-Weinberg equilibrium in the control group. COX-1 $22 \mathrm{C} / \mathrm{T}, \mathrm{COX}-25^{\prime} \mathrm{UTR} \mathrm{T} / \mathrm{G}, \mathrm{COX}-2$ Exon $10 \mathrm{~T} / \mathrm{C}$, and FLAP -336 G/A polymorphisms were significantly associated with CSU, carriers of the minor allele being more represented in the CSU group. Similar results were obtained comparing either the AtCSU or the AeCSU group with the NC group, respectively. On the other hand, no significant difference in genotype distribution was found between the AtCSU and AeCSU groups (table 2).

All the SNPs that were associated with CSU at univariate analysis were included in a sex- and age-adjusted multiple regression model. In that model only 3 SNPs, i.e. COX-2 5'UTR T/G, COX-2 Exon 10 T/C, and FLAP -336 G/A, remained significantly associated with $\mathrm{CSU}$, as shown in figure 1. Similar results were obtained as regards the specific association with AtCSU and AeCSU, even if the statistical significance of the association of COX-2 Exon 10 T/C polymorphism with AeCSU was marginally lost (figure 1). Moreover, in logistic regression analysis while all the 3 above mentioned polymorphisms remained significantly associated with CSU, no significant interaction among them in predicting CSU risk was found (table 3). Such results prompted us to develop an additive model considering the sum of effects of unfavourable, CSU-associated alleles.

Then, we evaluated the combined effects of these 3 SNPs and on the basis of them we constructed a genetic score, reflecting the sum of the concomitant "unfavourable" alleles (i.e. the alleles associated with CSU: COX-2 5'UTR G allele, COX-2 Exon $10 \mathrm{C}$ allele, and FLAP -336 G/A allele), theoretically ranging from 0 (no risk allele present) to 6 (all the risk alleles present). As shown in figure 2, the proportion of CSU patients increased progressively with the increasing number of unfavourable alleles $\left(P<0.001\right.$ by $\chi^{2}$-test for linear trend). Remarkably, all the subjects with more than 2 risk alleles had CSU and also in the group with 2 risk alleles, the great majority of subjects, i.e. $91.3 \%$ (21/23), presented CSU, whereas in the group with no risk allele the prevalence of CSU diagnosis was about the 30\% (37/122). Such results were substantially confirmed for both AtCSU and AeCSU (data not shown).

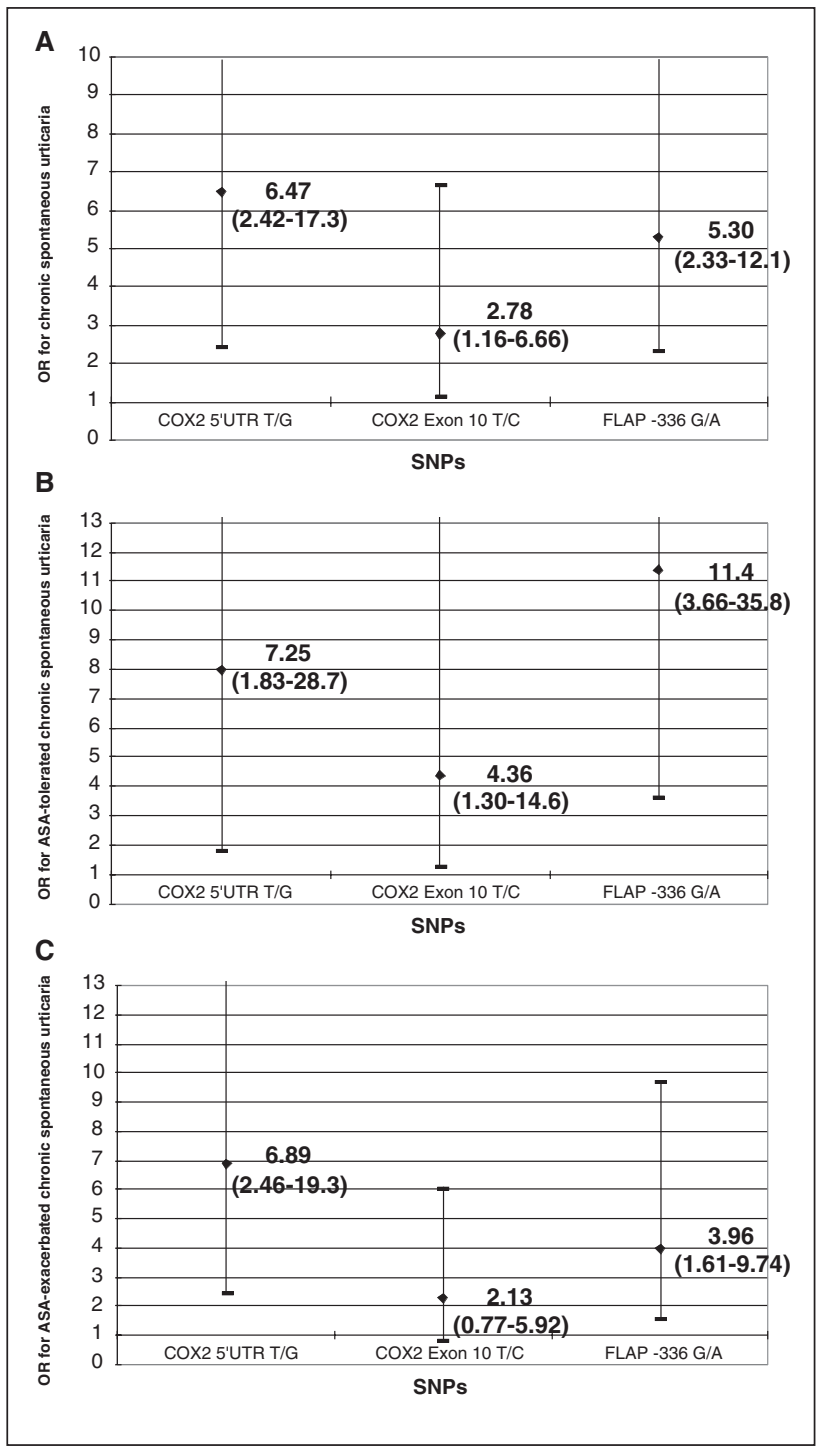

Figure 1. Odd Ratios (ORs) for chronic spontaneous urticaria (CSU) (A), ASA-tolerated CSU (B), and ASA-exacerbated CSU (C) of COX2 5'UTR G and Exon 10 C, and FLAP -336 A carriership in a multiple logistic regression, sex- and ageadjusted and including all the SNPs which were associated with CSU at the univariate analysis.

Finally, as regards the assessment of LTE4 levels before and after ASA challenge according to the different gene polymorphisms, no significant difference was found for LTE4 basal levels (data not shown). On the other hand,

Table 3. Multiple logistic regression considering COX1 and ALOX5 gene polymorphisms and their potential interaction as predictors of chronic spontaneous urticaria (CSU).

\begin{tabular}{|lrrr|}
\hline & B & Standard error & $P$ \\
\hline COX-2 5'UTR T/G & 1.558 & 0.461 & 0.001 \\
\hline COX-2 Exon 10 T/C & 1.615 & 0.541 & 0.003 \\
\hline FLAP -336 G/A & 1.018 & 0.483 & 0.035 \\
\hline COX-2 5'UTR T/G * COX-2 Exon 10 T/C & 19.158 & 17.008 & 0.999 \\
\hline COX-2 5'UTR T/G * FLAP -336 G/A & 18.808 & 11.832 & 0.999 \\
\hline COX-2 Exon 10 T/C * FLAP -336 G/A & 0.138 & 1.253 & 0.912 \\
\hline
\end{tabular}




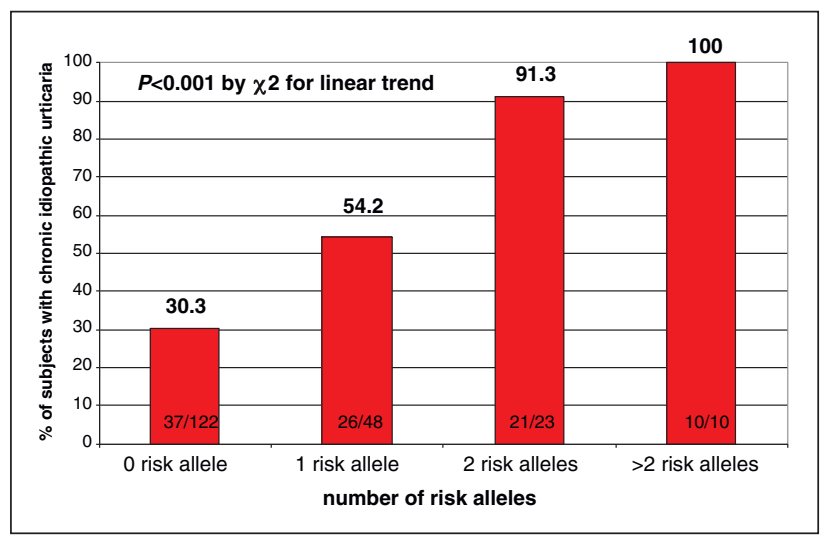

Figure 2. Prevalence of subjects with chronic spontaneous urticaria (CSU) according to a polygenic model constructed on the basis of the following three polymorphisms, i.e. COX2 5'UTR T/G and Exon $10 \mathrm{~T} / \mathrm{C}$, and FLAP -336 G/A. The COX2 $5^{\prime}$ UTR G and Exon $10 \mathrm{C}$, and FLAP -336 A alleles are considered as risk alleles.

Table 4. Predictors of post-ASA challenge LTE4 levels variability in linear regression model.

\begin{tabular}{|lcr|}
\hline & $\begin{array}{l}\text { Standardized } \\
\text { beta-coefficient }\end{array}$ & \multicolumn{1}{l|}{$\boldsymbol{P}$} \\
\hline Age (years) & -0.005 & 0.823 \\
\hline Male sex & -0.054 & 0.017 \\
\hline AtCSU & -0.006 & 0.808 \\
\hline AeCSU & 0.965 & $<0.001$ \\
\hline COX-1 22 T carriership & 0.072 & 0.001 \\
\hline COX-2 5'UTR G carriership & -0.026 & 0.271 \\
\hline
\end{tabular}

Adjusted $R^{2}=0.938$

COX-1 $22 \mathrm{~T}$ carriers $(52.5 \pm 31.1$ versus $39.1 \pm 26.9$ in CC homozygotes, $P=0.039$ by t-test) and COX-2 $5^{\prime}$ UTR G carriers $(54.0 \pm 26.4$ versus $38.3 \pm 27.9$ in TT homozygotes, $P=0.011$ by t-test) seemed to present higher LTE4 levels after ASA challenge. However, in a linear regression model after adjustment for disease status, only COX-1 $22 \mathrm{~T}$ carriership remained a significant predictor of post-challenge LTE4 levels variability (table 4). A further analysis showed that COX-1 $22 \mathrm{~T}$ carriers presented particularly high LTE4 levels after ASA challenge in the AeCSU group $(77.5 \pm 4.7$ versus $67.2 \pm 10.4$ in CC homozygotes, $P=0.001$ by t-test).

\section{Discussion}

Urticaria is a common skin disorder, classified as acute and chronic [13-16] Generally, in 40 to $60 \%$ of patients with chronic urticaria, the cause of chronic urticaria cannot be identified and in such cases, urticaria is defined as CSU. However, with the ingestion of ASA and/or NSAIDs and/or food additives, hypersensitivity can aggravate urticaria in these patients [13-17]. The choice of therapy in these patients, the particular subgroups of patients affected by
CSU with ASA or food additive hypersensitivity, is important, since not all respond to classic treatment (17) and the use of antileukotrienes has been suggested [9, 10, 18, 19]. However, it has been postulated that ASA sensitivity may be caused by an increased production of Cys-LT and a relative inhibition of COXs $[8,20]$, so that there would be a shift in the arachidonic acid metabolism away from the cyclooxygenase (COX) pathway toward the lipo-oxygenase (LO) pathway, which synthesized Cys-LTs (1). Our working hypothesis was that polymorphisms of these candidate genes could affect these pathways and, consequently, the risk of CSU and/or hypersensitivity to ASA. In the literature there are few studies that have assessed the potential role of candidate gene polymorphisms in CSU subjects. A previous study demonstrated that promoter polymorphisms of 5-LO (-1708 G/A) and CYSLTR1 (-634C/T) were significantly different between aspirin-intolerant asthma and aspirin-induced urticaria/angioedema, suggesting different contributions to the lipo-oxygenase pathway [21, 22].

This is the first study, to our knowledge, to investigate the possible influence of the polymorphisms of COXs and FLAP on CSU risk and on the levels of urinary leukotrienes, before and after oral challenge with ASA. We analyzed the polymorphisms of Cox-1, Cox-2, and FLAP in CSU patients and in controls from Northern Italy. We found that COX-2 5'UTR T/G, COX-2 Exon 10 T/C, and FLAP -336 G/A polymorphisms were associated with CSU. More precisely, a significant association was found for both AtCSU and AeCSU, whereas no significant difference in genotype distribution was found between AtCSU and AeCSU groups. Such results may suggest an ASA-independent link of the above mentioned polymorphisms with CSU.

Interestingly, we found also an additive effect of these SNPs on CSU risk. Of note, the use of a polygenic additive model in evaluating the combined effect of more risk alleles was further supported by the fact that no significant interaction among them in determining CSU risk was found. Indeed, the proportion of CSU patients increased progressively with increasing number of risk alleles, i.e. the alleles associated with CSU, and remarkably all the subjects with more than 2 risk alleles had CSU in our study-population. We are tempted to speculate that similar polygenic scores may be useful tools in future clinical practice, but these results certainly should be further investigated and replicated before drawing any kind of conclusion.

In addition, we evaluated the urinary LTE4 values, before and after ASA-challenge. Unfortunately, we do not have the data of urinary prostaglandins. COX $-122 \mathrm{~T}$ carriership was a significant predictor of post-challenge LTE4 level variability, suggesting a potential influence on the leukotriene pathway. However, COX-1 C/T was only slightly associated with either AtCSU or AeCSU at univariate analysis and, moreover, the statistical significance of this association was lost after adjustment in a multiple regression model. On the other hand, the CSU-associated COX-2 5'UTR G allele seemed to present higher LTE4 levels after ASA challenge, but the statistical significance of this association was lost after adjustment in the linear regression model for urinary LTE4 level variability. Such inconsistencies and disagreements in the results may also be related to the relatively low number of subjects enrolled and, consequently, to a lack of statistical power.

Certainly, the current study presents several limitations, mainly related to the case-control design and the relatively 
low sample size. Moreover, data about some other potential candidate polymorphisms, such as those of 5-LO, are lacking, as well as data about other markers of intermediate inflammatory pathways, like urinary prostaglandins. Therefore, our results should be considered only as hypothesis-generating. Nonetheless, our results suggest that genetic variants of candidate genes involved in prostaglandin and leukotriene metabolisms may be associated with CSU risk and CSU-related pathways. Further studies are needed to address such intriguing issues. In the future, such knowledge may lead to the development of new diagnostic markers and provide additional, genetic-tailored, therapeutic targets.

Disclosure. This study was supported by grants from MIUR (Italian University and Research Ministry) (former $60 \%$ funds) to Gabriele Di Lorenzo, Calogero Caruso and Roberto Corrocher. Vito Ditta is a PhD student of Pathobiology PhD course (directed by C.C.) at Palermo University and this work is submitted in partial fulfillment of the requirement for the PhD degree. No support was received from the pharmaceutical and diagnostic industry. The authors declare they have no competing of interest.

\section{References}

1. Hamad AM, Sutcliffe AM, Knox AJ. Aspirin-induced asthma: clinical aspects, pathogenesis and management. Drugs 2004; 64: 2417 . 32.

2. Zuberbier T, Asero R, Bindslev-Jensen C, et al. Dermatology Section of the European Academy of Allergology and Clinical Immunology; Global Allergy and Asthma European Network; European Dermatology Forum; World Allergy Organization. EAACI/GA(2)LEN/EDF/ WAO guideline: definition, classification and diagnosis of urticaria. Allergy 2009; 64: 1417-26.

3. Szczeklik A, Gryglewski RJ, Czerniawska-Mysik G. Clinical patterns of hypersensitivity to nonsteroidal anti-inflammatory drugs and their pathogenesis. J Allergy Clin Immunol 1977; 60: 276-84.

4. Pacor ML, Di Lorenzo G, Biasi D, Barbagallo M, Corrocher R. Safety of rofecoxib in subjects with a history of adverse cutaneous reactions to aspirin and/or non-steroidal anti-inflammatory drugs. Clin Exp Allergy 2002; 32: 397-400.

5. Zembowicz A, Mastalerz L, Setkowicz M, et al. Safety of cyclooxygenase 2 inhibitors and increased leukotriene synthesis in chronic idiophatic urticaria with sensitivity to non-steroidial anti-inflammatory drugs. Arch Dermatol 2003; 139: 1577-82.
6. Leone $S$, Ottani A, Bertolini A. Dual acting anti-inflammatory drugs. Curr Top Med Chem 2007; 7: 265-75.

7. Di Lorenzo G, Pacor ML, Vignola AM, Profita M, EspositoPellitteri $M$, Biasi $D$, et al. Urinary metabolites of histamine and leukotrienes before and after placebo-controlled challenge with ASA and food additives in chronic urticaria patients. Allergy 2002;57: $1180-6$.

8. Mastalerz L, Setkowicz M, Sanak M, Szczeklik A. Hypersensitivity to aspirin: common eicosanoid alterations in urticaria and asthma. $J$ Allergy Clin Immunol 2004; 113:771-5.

9. Pacor ML, Di Lorenzo G, Corrocher R. Efficacy of leukotriene receptor antagonist in chronic urticaria. A double-blind, placebo-controlled comparison of treatment with montelukast and cetirizine in patients with chronic urticaria with intolerance to food additive and/or acetylsalicylic acid. Clin Exp Allergy 2001; 31 : 1607-14.

10. Di Lorenzo G, Pacor ML, Mansueto $P$, et al. Randomized placebo-controlled trial comparing desloratadine and montelukast in monotherapy and desloratadine plus montelukast in combined therapy for chronic idiopathic urticaria. J Allergy Clin Immunol 2004; 114:619-25.

11. Titz NW. Fundamentals of Clinical Chemistry, 2nd ed.. Philadephia: WB Sauder, 1976, p. 994

12. Miller $S A$, Dykes $D D$, Polesky HF. A simple salting out procedure for extracting DNA from human nucleated cells. Nucleic Acid Res 1998; 16: 1215

13. Grattan CE. Aspirin sensitivity and urticaria. Clin Exp Dermatol 2003; 28: 123-7.

14. Grattan $C E$. The urticaria spectrum: recognition of clinical patterns can help management. Clin Exp Dermatol 2004; 29: 217-21.

15. Kröpfl L, Maurer M, Zuberbier T. Treatment strategies in urticaria. Expert Opin Pharmacother 2010; 1 1: 1445-50.

16. Fonacier L, Aquino $M$, Kim B. Clinical evaluation and treatment of chronic urticaria. Postgrad Med 2010; 122: 148-56.

17. Di Lorenzo G, Pacor ML, Mansueto P, Martinelli N, EspositoPellitteri $M$, Lo Bianco $C$, et al. Food-additive-induced urticaria: a survey of 838 patients with recurrent chronic idiopathic urticaria. Int Arch of Allergy Immunol 2005; 138: 235-42.

18. Di Lorenzo G, Pacor ML, Mansueto P, Esposito-Pellitteri M, Ditto $V$, Lo Bianco $C$, et al. Is there a role for antileukotrienes in urticaria? Clin Exp Dermatol 2006; 3: 327-34.

19. Bagenstose SE, Levin L, Bernstein JA. The addition of zafirlukast to cetirizine improves the treatment of chronic urticaria in patients with positive autologous serum skin test results. J Allergy Clin Immunol 2004; 113: 134-40.

20. Setkowicz M, Mastalerz L, Podolec-Rubis M, Sanak M, Szczeklik A. Clinical course and urinary eicosanoids in patients with aspirininduced urticaria followed up for 4 years. J Allergy Clin Immunol 2009; 123: 174-8.

21. Kim SH, Choi JH, Holloway JW, et al. Leukotriene-related gene polymorphisms in patients with aspirin-intolerant urticaria and aspirin-intolerant asthma: differing contributions of ALOX5 polymorphism in Korean population. J Korean Med Sci 2005; 20:92631.

22. Kim SH, Ye YM, Lee SK, Park HS. Genetic mechanism of aspirininduced urticaria/angioedema. Curr Opin Allergy Clin Immunol $2006 ; 6: 266-70$ 